\title{
Population Pharmacokinetic Parameter Estimates using a Limited Sampling Design: Analysis of Blood Alcohol Levels
}

\author{
Asuka Nemoto, Masaaki Matsuura, Kazue Yamaoka \\ Teikyo University Graduate School of Public Health, \\ 2-11-1 Kaga Itabashi-ku Tokyo 173-8605, Japan \\ *E-mail: anemoto@med.teikyo-u.ac.jp
}

(Received May 18; accepted November 1; published online November 30, 2016)

\begin{abstract}
When population pharmacokinetic (PPK) analysis is conducted, there are often constraints on obtaining blood samples at multiple time points. In this context, it is a concern that estimated PPK parameters are reported without an evaluation of their reliability. The aim of the present study was to improve the reliability of the estimated population mean pharmacokinetic parameters after assessing the precision and accuracy of the estimates by stochastic simulation and estimation. In an example of alcohol metabolism data, where samples taken later than 60 min after consumption were not available, the precision of the estimates for the population mean elimination rate "constant" $\left(k_{e l}\right)$ was found to be low. To tackle this problem, real-life data were aggregated with the data stochastically generated from historical knowledge, and the parameters were estimated. The estimated $k_{e l}$ value based on only real data was revealed to be biased toward a lower value.
\end{abstract}

Key Words: population pharmacokinetic analysis, estimation precision and bias, stochastic simulation, limited sampling design

Area of Interest: Information and computing approach for drug design and ADMET study 


\section{Introduction}

\subsection{Background}

Pharmacokinetics is the study of the time course of changing drug concentration over time [1]. A compartmental pharmacokinetic (PK) model describes and predicts the concentration-time profile assuming the body is divided into discrete compartments; thus, for example, the drug is modeled as distribute only to the blood in a one-compartment model, or to blood and tissues in a twocompartment model. The concentration at any given time point is modeled by a formula composed of PK parameters such as the absorption rate constant, the volume of distribution, the elimination constant and so on.

Population pharmacokinetics (PPK) is the study of the variability in drug concentration between individuals when the same or a standardized dose is administered [2-5]. Population analysis assumes the presence of inter-individual variations in PK parameters and attempts to account for this in terms of patient-specific covariates that could affect the PK model parameters, such as age, weight, disease state, and genotype.

It is important to examine the sampling design when obtaining data for PPK analysis. The major goal of most PPK studies is the precise and accurate estimation of PPK parameters. This is crucial because PK parameters are used, for example, for the calculation of the AUC, for calculating the duration time above the threshold, or in clinical trial simulations to model the physiological effects of the drug. Design factors that affect the accuracy of parameter estimates include the number of samples per subject, the arrangement of sampling points over the time course and the number of subjects to include in the study. Under circumstances in which researchers can control or influence the design of the study, there are two options. The first option is a simulation-based approach [6-14] and the second one is an information theory-based approach [3, 4, 15-17].

However, in real-world situations, it is uncommon to find that PK studies are performed according to an "informative" optimal design. Most PPK studies are performed as adjuncts to clinical trials [18]. Also, there may be several other constraints, such as ethical considerations as well as logistic impracticality, as in, for example, pediatric research [19]. Several solutions to this problem have been proposed thus far, such as using prior knowledge of the model parameters, including a Bayesian approach [20-22]. However, the range of applications appropriate for this approach is limited because these techniques assume that the PPK model used in the current analysis is the same as the model that was used in the earlier studies that are taken as the prior information. Unfortunately, the proposed methods so far are not applicable to the field of alcohol research. Looking at the history, there are accumulated results from the alcohol metabolism research in which time-concentration data were analyzed by the Widmark formula. The Widmark formula, which assumes that the elimination rate of blood alcohol is constant, is different from the compartment model, which has been commonly used in recent years and assumes that the elimination rate is dependent on the concentration. In this paper, we refer to the reliable estimates for the parameters of the Widmark formula as a "historical knowledge".

\subsection{Motivating Example}

A previous PPK study [23] analyzed blood alcohol concentration-time profiles using 157 samples taken from 34 healthy Japanese volunteers, consisting of 21 males and 13 females (mean age (SD), 29.4 (12.9) years; mean body weight (SD), $61.3(10.8) \mathrm{kg}$ ). One of the aims of the study was to identify covariates accounting for inter-individual variations in the alcohol absorption rate. We obtained blood samples only up to 60 min after drinking the alcohol because of imposed ethical 
limitations. Because food ingestion prior to alcohol consumption reduces peak blood alcohol concentrations and increases the rate of ethanol metabolism [24], so the subjects enrolled in our study received alcohol in the morning after an overnight fast. Due to the possible hypoglycemia and nausea caused by alcohol intake after overnight fasting and further considerations of subject stress, the latest sampling time point was 60 min after alcohol intake and the number of samples per subject was limited to a maximum of five.

As the base population pharmacokinetic model of choice, a one-compartment model with a normal first-order elimination process was selected because it described our blood alcohol concentration data well. Though the blood alcohol elimination process is known to follow MichaelisMenten kinetics, at low concentrations of alcohol, this is known to be almost equal to a first-order elimination process $(<400 \mathrm{mg} / \mathrm{L})$ [25]. This made the selected base model simpler, with a reduced number of parameters to be estimated. Patient characteristics tested as potential covariates of a nonlinear mixed effect model were age, gender, body weight, and alcohol metabolism-related gene polymorphisms. The model building process consisted of two sequential steps. For this, a generalized additive modelling (GAM) analysis, using Akaike's information criterion [5] was applied. As a first step, the relationships between the PK parameters estimated for the individual subject and the candidate covariates were tested using F statistics. As a second step, each variable that was positive in the first step was inserted into the model as an independent variable for the dependent variable of the natural log of the related PK parameter. The variable associated with the highest AIC reduction was then incorporated into the model. The candidate variables were incorporated one by one in a forward selection manner until the decline of the AIC ceased.

Estimates of the population PK parameters in the final model are depicted in Table 1 . We considered that the appropriate interpretation about the reliability for estimates of the parameters is required in order to assist the reader. Particularly, there was a concern about the reliability of estimates of the population mean elimination rate constant $k_{e l}$, because our data consists of only the early part of the time-concentration curve.

\subsection{Study Aim}

In this paper, we refer to a study sampling design of the type given in the motivating example above as a "limited sampling design" (LS design) study, where no samples are available after a certain time. We refer to a "rich" and extensive sampling design, which has been employed in traditional PK studies, as a "dense sampling design" (DS design).

The aim of the present study was to evaluate the precision and accuracy of PPK parameters estimated by fitting the model to the simulated data from the LS design study, compared with the DS design, and subsequently to propose a procedure that enables estimation of the direction and magnitude of the estimation bias by using historical knowledge.

\section{Methods}

First, we will provide a description of the PPK model and the procedure for stochastic simulation and estimation. Second, we provide a description of the procedure by which we estimated the direction and magnitude of the bias of the estimate of one of the parameters, where we found problems in reliability. All statistical analyses and simulation experiments were performed with SAS version 9.3 (SAS Institute Inc., Cary, NC, USA.). 
Table 1. Population Pharmacokinetic Parameters for Ethanol ${ }^{\mathrm{a}}$

${ }^{a}$ Reprinted from table 3.2 [23]. CI: confidence interval.

\begin{tabular}{|c|c|c|c|}
\hline Parameter & Estimate & \multicolumn{2}{|c|}{$95 \% \mathrm{CI}$} \\
\hline \multicolumn{4}{|l|}{ Fixed Effect } \\
\hline$V_{d} / F, \mathrm{~L}$ & 51.2 & 37.4 & 69.9 \\
\hline$\theta_{A G E\left(V_{d} / F\right)}, \log (\mathrm{L})$ & -0.017 & -0.028 & -0.006 \\
\hline$\theta_{W E I G H T\left(V_{d} / F\right)}, \log (\mathrm{L})$ & 0.013 & 0.005 & 0.023 \\
\hline$k_{a}, \min ^{-1}$ & 0.047 & 0.033 & 0.069 \\
\hline$\theta_{A G E\left(k_{a}\right)}, \log (\mathrm{L})$ & 0.045 & 0.027 & 0.063 \\
\hline$k_{e l}, \min ^{-1}$ & 0.014 & 0.009 & 0.022 \\
\hline \multicolumn{4}{|c|}{ Inter-individual Variation } \\
\hline$\sigma_{V_{d} / F}, \log (\mathrm{L})$ & 0.286 & 0.121 & 0.452 \\
\hline$\sigma_{k_{a}}, \log \left(\mathrm{min}^{-1}\right)$ & 0.601 & 0.39 & 0.813 \\
\hline \multicolumn{4}{|c|}{ Intra-individual Variation } \\
\hline$\sigma, \mathrm{g} \mathrm{L}^{-1}$ & 0.204 & 0.162 & 0.257 \\
\hline \multicolumn{4}{|c|}{$\begin{array}{l}\text { Structural mode } \\
\quad \begin{array}{l}\log \left(V_{d} / F_{i}\right)=\log \left(V_{d} / F\right)+\theta_{A G E\left(V_{d} / F\right)} \times\left(A G E_{i}-30\right)+\theta_{W E I G H T\left(V_{d} / F\right)} \\
\quad \times\left(W E I G H T_{i}-60\right)+\eta_{V_{d} / F_{i}}\end{array}\end{array}$} \\
\hline \multicolumn{4}{|c|}{$\log k_{a_{i}}=\log k_{a}+\theta_{A G E\left(k_{a}\right)} \times\left(A G E_{i}-30\right)+\eta_{k_{a_{i}}}$} \\
\hline
\end{tabular}

\subsection{Stochastic Simulation and Estimation}

\subsubsection{Data Simulation and Sampling Design}

The objective of the data simulations was to compare the precision and accuracy between two sampling designs in estimating population mean PK parameters. Blood alcohol concentrations after alcohol intake were simulated using a one-compartment model and first-order absorption according to equation (1),

$$
C_{i j}=\frac{D_{i} \cdot k_{a_{i}}}{V_{d} / F_{i} \cdot\left(k_{a_{i}}-k_{e l_{i}}\right)}\left\{\exp \left(-k_{e l_{i}} \cdot t_{i j}\right)-\exp \left(-k_{a_{i}} \cdot t_{i j}\right)\right\}
$$

where $V_{d} / F_{i}, k_{a_{i}}$ and $k_{e l_{i}}$ are the individual parameters for the ith individual; $C_{i j}$ is the modelpredicted concentration in the $i$ th individual at the $j$ th time point $t_{i j}$; and $D_{i}$ is the amount of alcohol consumed by the $i$ th individual.

Individual values for each parameter were obtained according to condition (2),

$$
\begin{gathered}
\boldsymbol{\theta}_{i}=\left(\begin{array}{c}
V_{d} / F_{i} \\
k_{a_{i}} \\
k_{e l_{i}}
\end{array}\right), \quad \boldsymbol{\mu}_{\theta}=\left(\begin{array}{c}
V_{d} / F \\
k_{a} \\
k_{e l}
\end{array}\right) \\
\log \boldsymbol{\theta}_{i} \sim N\left(\log \boldsymbol{\mu}_{\boldsymbol{\theta}}, \boldsymbol{\Sigma}\right), \quad \boldsymbol{\Sigma}=\left(\begin{array}{ccc}
\sigma_{V_{d} / F^{2}} & 0 & 0 \\
0 & \sigma_{k_{a}}{ }^{2} & 0 \\
0 & 0 & \sigma_{k_{e l}}{ }^{2}
\end{array}\right)
\end{gathered}
$$


where $V_{d} / F$ is the population mean of the apparent volume of distribution, $k_{a}$ is the population mean of the absorption rate "constant", $k_{e l}$ is the population mean of the elimination rate "constant" and $\mathrm{N}$ represents the normal distribution. Individual parameters were assumed to follow a log-normal distribution, where $\sigma_{V_{d} / F}{ }^{2}, \sigma_{k a}{ }^{2}$ and $\sigma_{k e l}{ }^{2}$ are inter-individual variations of each of the apparent volumes of distribution, the absorption rate and the elimination rate.

The residual errors between the model-predicted and observed concentrations were assumed to be a proportional model, as shown in equation (3),

$$
y_{i j}=C_{i j} \times\left(1+\varepsilon_{i j}\right), \quad \varepsilon_{i j} \sim \mathrm{N}\left(0, \sigma^{2}\right)
$$

where $y_{i j}$ is the observed concentration for the $i$ th individual at the $j$ th time point.

To simulate the LS design data, modeling was performed according to equation (1). The condition of the simulation was that each of the 34 subjects consumed a single dose of $17.5 \mathrm{~g}$ of alcohol and observations were obtained at 5, 10, 20, 30 and 60 min thereafter. To simulate the DS design data, the model, alcohol dose and number of subjects were set as the same as for the LS design, except that there were 8 sampling times as at 5, 10, 20, 30, 60, 120, 180, and $240 \mathrm{~min}$ post-dose. Table 1 shows the values of the parameters for each simulation setting. The parameters for Case 1, the first simulation scenario, were determined by referencing the estimates of the motivating example summarized in Table 2.

Table 2. Population Pharmacokinetic Parameters in the Simulation Study The parameters for Case 1 were determined by referencing the estimates of the motivating example summarized in Table 2. $V_{d} / F$ is set to be twice in Case 2, and is set to be half in Case3.

\begin{tabular}{|c|c|c|c|c|}
\hline Parameter & Case 1 & Case 2 & Case 3 & Case 4 \\
\hline \multicolumn{5}{|l|}{ Fixed Effect } \\
\hline$V_{d} / F, \mathrm{~L}$ & 45 & 90 & 22.5 & 45 \\
\hline$k_{a}, \min ^{-1}$ & 0.1 & 0.1 & 0.1 & 0.1 \\
\hline$k_{e l}, \min ^{-1}$ & 0.01 & 0.01 & 0.01 & 0.01 \\
\hline \multicolumn{5}{|c|}{ Inter-individual Variation } \\
\hline$\sigma_{V_{d} / F}, \log (\mathrm{L})$ & 0.4 & 0.4 & 0.4 & 0.1 \\
\hline$\sigma_{k_{a}}, \log \left(\min ^{-1}\right)$ & 0.4 & 0.4 & 0.4 & 0.1 \\
\hline$\sigma_{k_{e l}}, \log \left(\min ^{-1}\right)$ & 0.4 & 0.4 & 0.4 & 0.1 \\
\hline \multicolumn{5}{|c|}{ Inter-individual Variation } \\
\hline$\sigma, \mathrm{g} \mathrm{L}^{-1}$ & 0.2 & 0.2 & 0.2 & 0.2 \\
\hline
\end{tabular}

Simulation settings other than those for Case 1 were set to determine whether slight differences in pharmacokinetics caused a different result from that obtained in Case $1 . V_{d} / F$ is set to be twice as high in Case 2, and is set to be half in Case 3. Inter-individual variability of population mean PK parameters were set to be smaller in Case 4. To illustrate the two sampling designs, one set of simulated data from Case 1 is shown in Figure 1. 

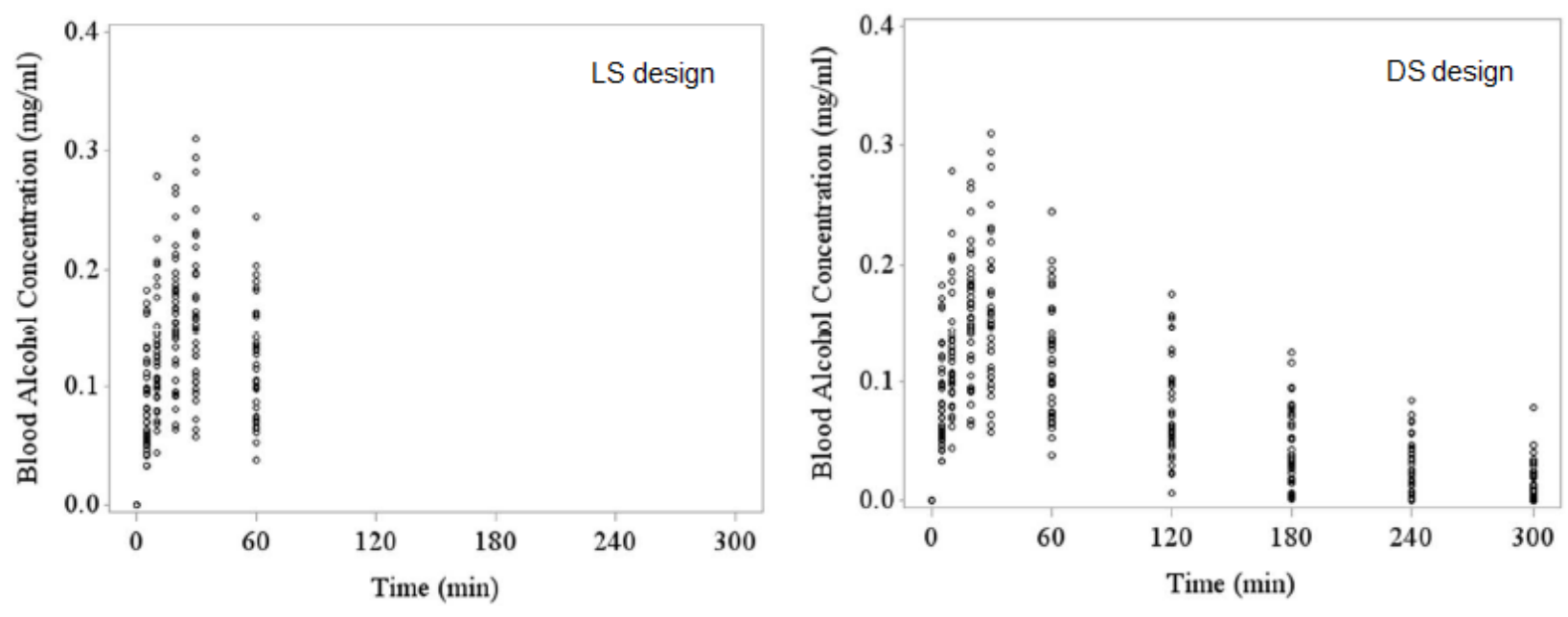

Figure 1. Sampling Time Points for LS and DS designs in the Simulation One set of simulated data is presented for each sampling design. Limited sampling design (LS design); dense sampling design (DS design).

\subsubsection{Estimation of Parameters and Evaluation of Precision and Accuracy}

Population PK parameters were estimated using the same analytical model as the model that simulated the data shown in equation (1). Analysis of each replicated dataset was facilitated by the NLMIXED procedure in SAS 9.3. Because the likelihood function integrated over the random effects does not have a closed-form expression, adaptive Gaussian quadrature was used to approximate likelihoods, and a dual quasi-Newton algorithm was used as a maximization technique. Bias in estimates of PK parameters for each NLMIXED run were calculated according to equation (4). The effects of sampling design on the precision and accuracy of estimation were investigated by obtaining the 2.5th percentile, median, and 97.5 th percentile of $\% \operatorname{bias}\left(\hat{\mu}_{\theta_{k}}\right)$,

$$
\% \operatorname{bias}\left(\hat{\mu}_{\theta_{k}}\right)=\frac{\widehat{\mu}_{\theta_{k}}-\mu_{\theta}}{\mu_{\theta}} \times 100,(\%)
$$

where the mean PK parameter $\theta$ is expressed as $\mu_{\theta}$, and the estimate of the $k$ th data set as $\hat{\mu}_{\theta_{k}}$.

\subsection{Estimation of the Bias using Historical Knowledge}

\subsubsection{Data Simulation Using Historical Knowledge}

Accurate understanding of alcohol kinetics has important implications in forensic science. For example, the quantity of alcohol consumed within a specific time-frame is valuable information when interviewing suspected drunk drivers. In forensic science, Widmark's method [27] has been widely used to convert blood alcohol concentrations into the amount of alcohol consumed,

$$
C^{w}{ }_{i j}=\frac{D_{i}}{\rho_{i} \times W E I G H T_{i}}-\beta_{i} \times t_{i j}+\varepsilon_{i j}, \quad \varepsilon_{i j} \sim N\left(0, \sigma^{2}\right)
$$

where $C^{w}{ }_{i j}$ is the concentration in the $i$ th individual at the $j$ th time point $t_{i j}, D_{i}$ is the volume of alcohol consumed, $\rho_{i}$ is the volume of distribution per body weight, $\beta_{i}$ is the zero-order 
elimination rate of alcohol from the blood, and WEIGHT is the weight for the $i$ th individual (equation (5)).

Individual values for parameters were generated according to condition (6),

$$
\boldsymbol{\theta}_{i}=\left(\begin{array}{c}
\rho_{i} \\
\beta_{i}
\end{array}\right), \quad \boldsymbol{\mu}_{\theta}=\left(\begin{array}{l}
\rho \\
\beta
\end{array}\right), \quad \boldsymbol{\theta}_{i} \sim N\left(\boldsymbol{\mu}_{\boldsymbol{\theta}}, \boldsymbol{\Sigma}\right), \quad \boldsymbol{\Sigma}=\left(\begin{array}{cc}
\sigma_{\rho}^{2} & 0 \\
0 & \sigma_{\beta}^{2}
\end{array}\right)
$$

where $\rho$ is the population mean of the volume of distribution per body weight, $\beta$ is the population mean of the zero-order elimination rate of alcohol from the blood and $\mathrm{N}$ represents the normal distribution. Individual parameters were assumed to follow a log-normal distribution, where $\sigma_{\rho}{ }^{2}$ and $\sigma_{\beta}^{2}$ are inter-individual variations of each of the apparent volumes of distribution, the absorption rate and the elimination rate.

Parameter values for generating data are as follows: $\rho=0.70 \mathrm{~L} / \mathrm{kg} ; \sigma_{\rho}=0.07 \mathrm{~L} / \mathrm{kg} ; \beta=0.140$ $\mathrm{g} / \mathrm{L} / \mathrm{hr} ; \sigma_{\beta}=0.02 \mathrm{~g} / \mathrm{L} / \mathrm{hr} ;$ mean body weight $=60 \mathrm{~kg}$ (standard deviation $=10 \mathrm{~kg}$ ); $\sigma=0.036 \mathrm{~g} / \mathrm{L}$. These values were determined with reference to estimated constants of Widmark's formula, which have been reported since the 1990s (Table 3) [28-32].

Table 3. Reported Parameter Estimates using Widmark's Formula -: Not reported. SD: standard deviation.

\begin{tabular}{lllc}
\hline & $\begin{array}{c}\text { Subject } \\
\mathrm{M} / \mathrm{F}\end{array}$ & $\begin{array}{c}\text { Mean } \rho \text { (SD) } \\
\mathrm{L} \mathrm{kg}^{-1}\end{array}$ & $\begin{array}{c}\text { Mean } \beta \text { (SD) } \\
\mathrm{g} \mathrm{L}^{-1} \mathrm{hr}^{-1}\end{array}$ \\
\hline Yamamoto et al., 1993 & $93 \mathrm{M}$ & - & $0.136(0.020)$ \\
Gullberg et al., 1994 & $108 \mathrm{M}$ & $0.69(0.061)$ & $0.133(0.020)$ \\
Nishimura et al., 2006 & $151 \mathrm{M}$ & - & $0.144(0.020)$ \\
Gullberg et al., 2007 & $24 \mathrm{M}$ & $0.73(0.067)$ & $0.148(0.032)$ \\
Dettling et al., 2007 & $68 \mathrm{M}$ & - & $0.158(0.028)$ \\
& $64 \mathrm{~F}$ & - & $0.179(0.030)$ \\
\hline
\end{tabular}

\subsubsection{Parameter Estimation Using Data Complemented by Historical Knowledge}

We attempted to create data complemented on the basis of historical knowledge. The small datasets composed of 34 observations from 34 subjects (one observation per subject) simulated 500 times with varying ranges of sampling times were combined with the real datas of the motivating example (a total of 157 observations from 34 subjects) to estimate parameters of the PPK model. The small dataset generated by Widmark's formula does not have information on age but the PPK model of the example case requires age as a covariate. Therefore, the mean age of subjects used in the motivating example was uniformly employed in the simulation. Data were simulated in 3 patterns with different sampling time ranges, as follows: 60-120 min (Pattern 1), 60-180 min (Pattern 2), and 60-240 min (Pattern 3). To illustrate the method used to create complemented data, examples of the combined data set for each pattern are shown in Figure 2. 

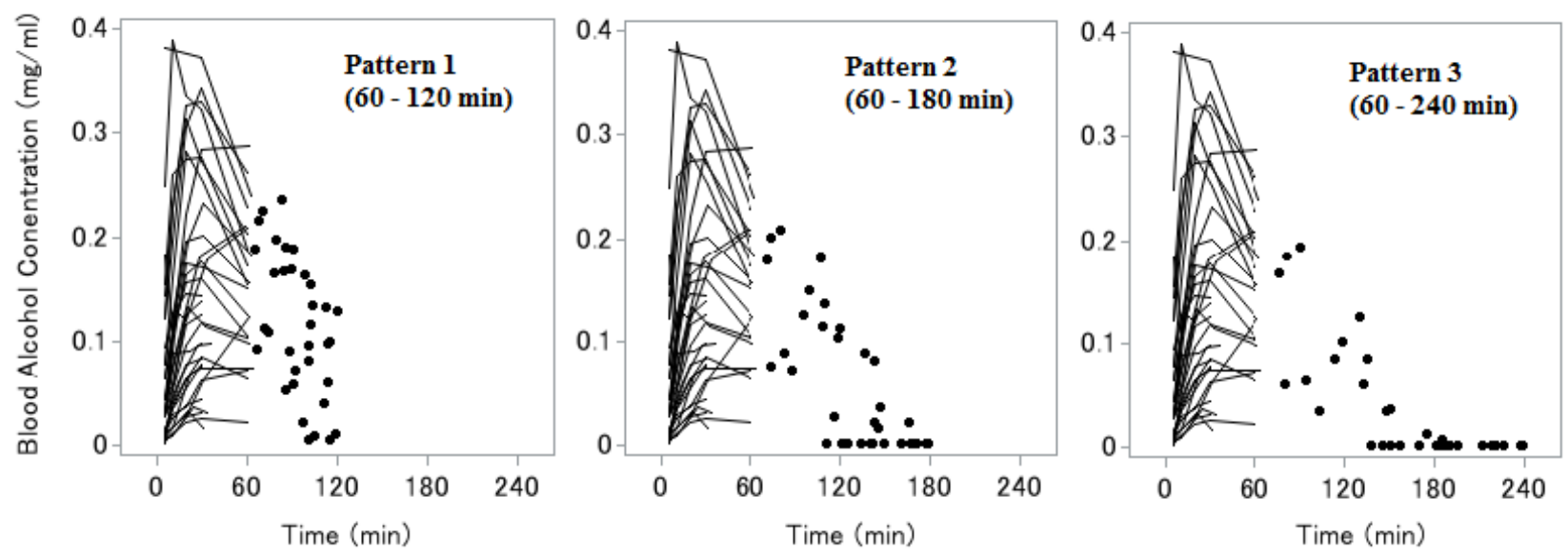

Figure 2. Patterns of Complemented Data

One set of simulated data for each pattern is presented. Real data are presented as unbroken lines and complemented data as dotted lines.

\section{Results}

\subsection{Estimation Bias of Population Mean PK Parameters in the LS Design}

The distribution of \%bias of estimates of population mean PK parameters is numerically examined here. The distribution of \%bias of estimates in the simulation setting of Case 1 is displayed as a box-and-whisker plot (Figure 3), revealing poor precision for the LS design relative to the DS design, albeit only for $k_{e l}$. Table 4 presents the $95 \%$ distribution range of \%bias for population mean PK parameters $\left(V_{d} / F, k_{a}\right.$ and $\left.k_{e l}\right)$ for the LS design and the DS design. The $95 \%$ range of \%bias of $k_{e l}$ for the LS design $(-40.7 \%,+51.0 \%)$ was approximately 3 -fold greater than for the DS design $(-12.7 \%,+15.2 \%)$, whereas the $95 \%$ range of \%bias for $V_{d} / F$ and $k_{a}$ did not differ significantly between the two. The lack of precision in estimates of $k_{e l}$ with the LS design in Case 1, as shown in Figure 3, was seen in all other simulation settings (Table 4). The convergence rate of an iterative maximization of an approximated likelihood for each Case was at least $73 \%$, which was high enough and generally acceptable. Though the convergence rates for DS design were relatively low (footnotes of Table 4), there were no remarkable differences in distribution of the estimated population mean PK parameters between LS design and DS design.

Regardless of sampling design, with all population mean PK parameters the median \%bias was approximately 0 , indicating that the LS design did not cause estimation bias.

\subsection{Estimation of the Bias using Historical Knowledge}

Results from all sampling time ranges (Pattern 1 to Pattern 3) yielded median $k_{e l}$ estimates that were higher than the estimate in the motivating example [23], although convergence rates were low (approximately 10\% in Patterns 1 and 2 and 30\% in Patterns 3). The estimate of $k_{e l}$ in the motivating example was 0.014 (Table 1), 34.6\% lower than the median values of 0.021 for Pattern 3 (Figure 4). For every pattern, the medians of estimates of $k_{e l}$ were greater than the estimate of $k_{e l}(0.0140)$ in the motivating example that is presented as a reference. This implies that there was an estimation bias toward lower values caused by the LS design. These results are consistent with the range of \%bias 
for $k_{e l}$ in Table 4.

To reveal the influence of the bias of $k_{e l}$ values, the calculated time-course of blood alcohol concentrations is presented in Figure 5. For the curve shown as a solid line, we used the values for $k_{e l}, V_{d} / F$ and $k_{a}$ that were obtained in the previous analysis. Assuming the $k_{e l}$ value to be $30 \%$ greater than the value of the solid line, the curve shown as a dotted line was calculated using the estimated value obtained by dividing the $k_{e l}$ estimated in the previous study by 0.7 . The AUC calculated for the dotted line plot was $28 \%$ less than that for the solid line.

Table 4. Comparison of the Bias and Precision of Parameter Estimation Between Limited and Dense Sampling Designs

The parameters for Case 1 were determined by referencing the estimates of the motivating example summarized in Table 2. $V_{d} / F$ is set to be twice in Case 2, and is set to be half in Case3. Convergence rate $=$ (the number of data set in which iteration converged) / (the number of the total data set). ${ }^{a}$ Convergence rates were $81.6 \%$ for LS design and $57.4 \%$ for DS design. ${ }^{b}$ Convergence rates were $73.8 \%$ for LS design and $68.0 \%$ for DS design. ${ }^{c}$ Convergence rates were $87.2 \%$ for LS design and $59.0 \%$ for DS design. ${ }^{\mathrm{d}}$ Convergence rates were $90.6 \%$ for LS design and $64.2 \%$ for DS design. LS design: limited sampling design; DS design: dense sampling design.

\begin{tabular}{|c|c|c|c|c|c|c|c|}
\hline & & \multicolumn{6}{|c|}{ \%bias (\%) } \\
\hline & & \multicolumn{2}{|c|}{$k_{a}$} & \multicolumn{2}{|c|}{$V_{d} / F$} & \multicolumn{2}{|c|}{$k_{e l}$} \\
\hline & & LS design & DS design & LS design & DS design & LS design & DS design \\
\hline \multirow[t]{3}{*}{ Case $1^{\mathrm{a}}$} & 97.5 percentile & 27.6 & 19.0 & 21.6 & 13.9 & 51.0 & 15.2 \\
\hline & Median & -1.4 & 0.8 & -0.3 & 0.6 & 2.2 & 0.1 \\
\hline & 2.5 percentile & -25.8 & -15.2 & -19.1 & -14.7 & -40.7 & -12.7 \\
\hline \multirow[t]{3}{*}{ Case $2^{b}$} & 97.5 percentile & 27.3 & 15.5 & 21.6 & 15.6 & 47.2 & 13.4 \\
\hline & Median & 1.5 & -0.5 & 0.7 & -0.2 & 0.7 & -0.6 \\
\hline & 2.5 percentile & -22.4 & -21.1 & -18.8 & -30.5 & -40.5 & -17.5 \\
\hline \multirow[t]{3}{*}{ Case $3^{c}$} & 97.5 percentile & 28.9 & 16.6 & 23.8 & 13.3 & 94.1 & 17.5 \\
\hline & Median & 0.1 & -0.2 & 0.8 & 0.3 & 0.6 & -0.3 \\
\hline & 2.5 percentile & -32 & -16.7 & -25.3 & -14.2 & -93.5 & -15.0 \\
\hline \multirow[t]{3}{*}{ Case $4^{d}$} & 97.5 percentile & 22.5 & 10.3 & 14.2 & 6.0 & 49.0 & 4.7 \\
\hline & Median & -1.2 & 0.9 & -0.3 & 0.1 & 1.8 & 0.0 \\
\hline & 2.5 percentile & -21.7 & -10.5 & -15.8 & -7.2 & -34.3 & -7.0 \\
\hline
\end{tabular}

\section{Discussion}

In the present study, we evaluated the accuracy of population mean PK parameters which were estimated using data obtained from an LS design study. Stochastic simulation and estimation revealed that the estimated population mean $k_{e l}$ from the LS design could be inaccurate, while there were not problems in the estimation accuracy of other population mean PK parameters. Complemented data based on the historical knowledge led to the suggestion that PK parameters were underestimated by about $30 \%$ in the previous analysis. 


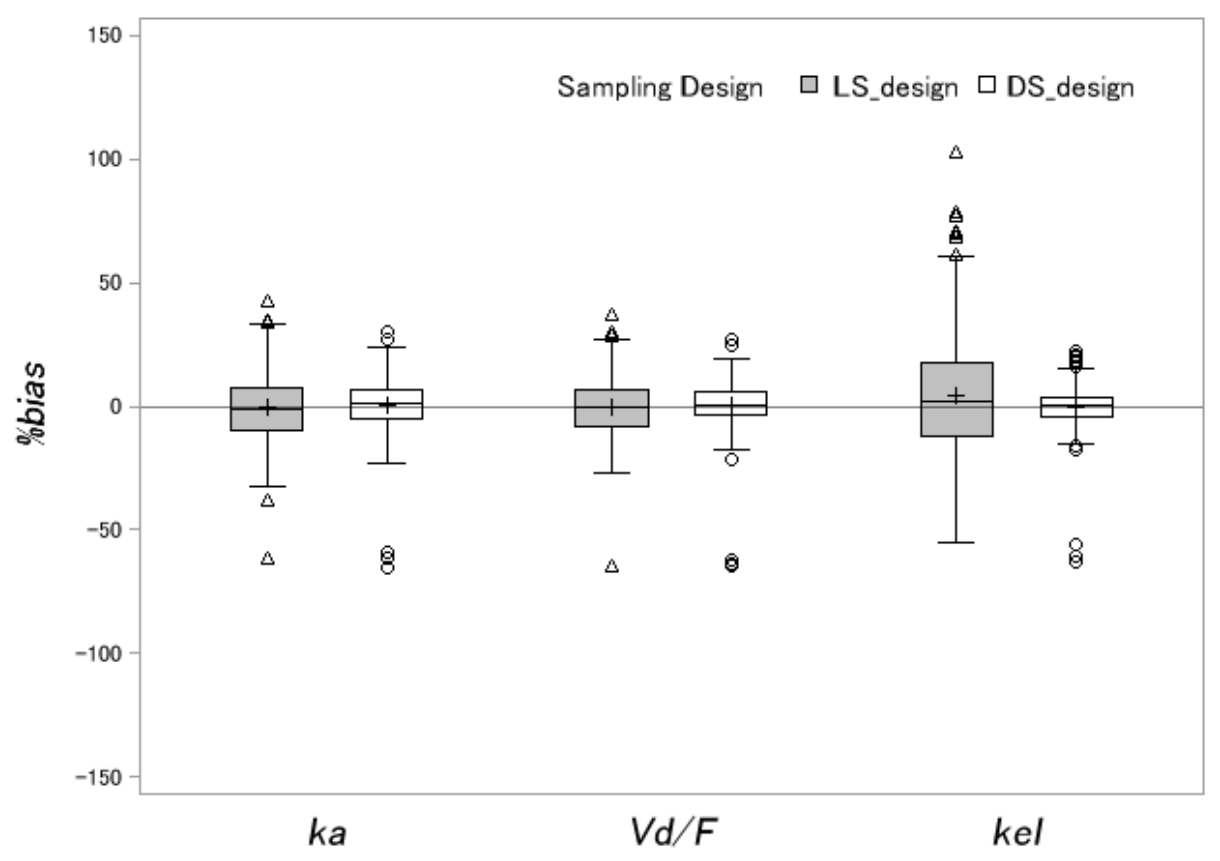

Figure 3. Distribution of \%bias of Population Pharmacokinetic Parameter Estimates in the Simulation Setting of Case 1

The gray filled boxes indicate the LS design; the open boxes the DS design. The precision of the estimated $k_{e l}$ was poorer in the LS than DS design study. $V_{d} / F$ is the population mean of the apparent volume of distribution, $k_{a}$ is the population mean of the absorption rate constant, and $k_{e l}$ is population mean of the elimination rate constant. The centerline and vertical ends of the boxes represent the median, 25th, and 75th percentile of the distributions, and the whiskers extend to the full range of the data or to 1.5 -fold of the interquartile range (IQR) from the median value, whichever was less. The \%bias outside of 1.5-fold of the IQR are plotted as triangles for the LS design and circles for the DS.

We proposed the method by which the magnitude and the direction of the bias of estimated PPK parameters in LS design studies can be estimated, regardless of the differences in models between the current analysis and the previously reported analyses. In reality, most population PK data are obtained in adjunct trials. Therefore, it is often difficult to obtain samples according to a preferred time schedule. In Phase III clinical trials, the main objective is to demonstrate drug efficacy and safety. In such context, study design (e.g. the number of samples per patient, timing of sampling, or the number of samples) is rarely based on the optimal sampling design best for PK analysis, but instead, is based on constraints imposed by the primary objective of the clinical trial $[18,32]$. As a practical measure to such a situation, we considered that the results of population PK analysis should be accompanied by an evaluation of the reliability of parameter estimates and an assessment of the magnitude and the direction of the bias. Some statistical methods including Bayesian modeling have been proposed to incorporate prior information into the current estimation of population PK parameter [21, 22, 33]. However, these techniques are not applicable to our case: the historical knowledge was obtained by using Widmark formula that is different from the analysis model, the compartment model. In contrast, our proposed method can be adopted regardless of the difference in models between the current and previous analysis. 


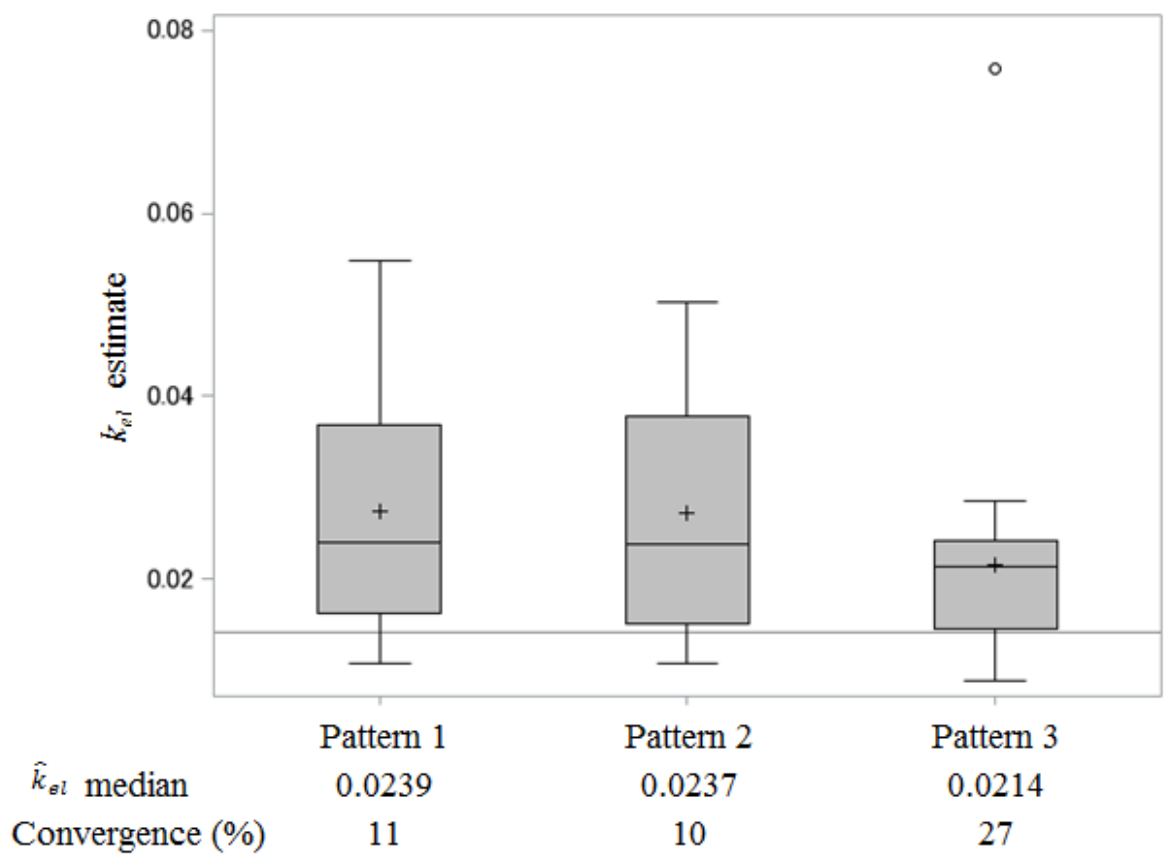

Figure 4. Distribution of $k_{e l}$ Estimates for Each Complemented Pattern

In every pattern, the median of estimates of $k_{e l}$ was greater than the estimate of $k_{e l}(0.0140)$ in the motivating example that is presented as a reference line. The centerline and vertical ends of the boxes represent the median, 25th, and 75th percentile of the distributions, and the whiskers extend to the full range of the data or to 1.5 -fold of the interquartile range from the median, whichever was less.

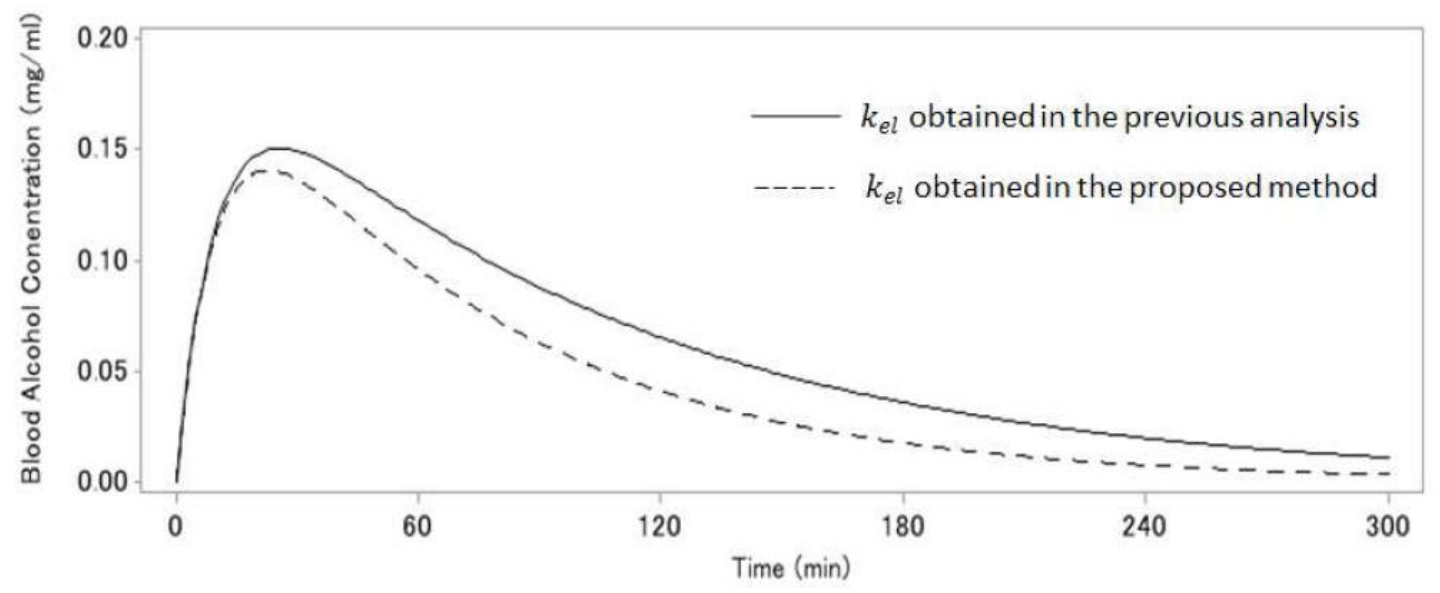

Figure 5. Graphic Presentation of the Influence of Bias in $k_{e l}$ Estimates on the Area Under the Time-concentration Curve

The solid line indicates the time-course of blood alcohol concentrations calculated using the estimates of population mean pharmacokinetic parameters that were obtained in the previous analysis. Dotted lines indicate the calculated blood alcohol concentration using the estimate of $k_{e l}$ obtained by using the proposed method.

Our idea is based on the knowledge that the concentrations obtained from the late part of the timeconcentration curve are crucial in order to accurately estimate $k_{e l}$. Norberg et al. illustrated that the 
late phase of time-concentration curve is "information-rich" with respect to PK parameters related to the elimination process, by showing the plot of absolute values of partial derivative of predicted concentration with respect to PK parameters related to the elimination were high in the late part of time-concentration curve [34]. This means that the late phase of time-concentration curve is "information-rich" with respect to $k_{e l}$. We considered that the estimation accuracy would be greatly improved, if observations, that were virtually obtained from the late part of the time-concentration curve, were added to a real dataset. And also, we considered that the magnitude and the direction of the bias of the estimates of $k_{e l}$ obtained from LS design can be assessed by using such dataset, which was referred as complemented data. The requirement of the data-generating model was considered to be the one that uses the historical knowledge obtained from the accumulated experimental results. Thus, we chose Widmark formula as a data-generating model, although this is not a compartment model.

As a sensitivity analysis, the additional simulation study was conducted using the population pharmacokinetic model, which was recently reported by Lee et al. [35], as a data-generating model. The basic model of Lee's analysis was 1-compartment model with Michaelis-Menten elimination process. We considered that this would be effective to dispel a possible doubt for using linear regression model (i.e. Widmark formula) as a data-generating model, though the weak point is that the parameter estimates are based on a single report. Lee's PPK model was used for the simulation study of Fig. 4, instead of Widmark formula. As a result, the conclusion was essentially the same. That is, the distribution of the estimated elimination rate constant values for complemented datasets generated by using the population pharmacokinetic model reported by Lee et al. was shifted to a higher side (each median of the distribution of estimated $k_{e l}$ for Pattern 1, Pattern 2 and Pattern 3, was $0.023,0.037$ and 0.037 , respectively), suggesting that the previously reported estimate for elimination rate constant by LS design was biased to a smaller value.

In this paper, the estimation accuracy of population mean PK parameters was presented by the distribution of the estimated values (i.e. the $95 \%$ range and the median value) obtained from a stochastic simulation and evaluation. The importance of simulation studies in assessing the impact of the study design on estimation accuracy has been pointed out [36]. Indeed, simulation studies intended to compare proposed study designs have yielded many findings concerning experimental designs for obtaining relatively accurate and precise parameter estimates [6-11]. In the given study design, Meibohm et al. have proposed to use the simulation study in order to address limitation of the LS design [19]. As shown in Table 4, the 95\% range of the estimated values of $k_{e l}$ was $(-40.7 \%$, $51.0 \%)$. Such bias influences greatly on the estimated area under the time-concentration curve. For instance, the influence of $30 \%$ difference in the $k_{e l}$ value on the area under the time-concentration curve was depicted in Fig. 5.

For generalization of the results in terms of the evaluation of the reliability of parameter estimates, in addition to the conditions presented in Table 2, we also conducted simulations under conditions with different $k_{e l}$ values. The 95\% range of \%bias of $k_{e l}$ from the LS design and DS design with a half value of $k_{e l}$ of Case 1 , were $(-93.4 \%, 91.2 \%)$ and $(-18.3 \%, 17.1 \%)$, respectively. This was similar to the result obtained from Case 1 . However, in terms of twice $k_{e l}$ of Case 1 , the reliable results were not obtained due to a low convergence rate in DS design. With twice the value of $k_{e l}$, convergence rates were $90.8 \%$ for LS design and $10.8 \%$ for DS design; with a half value of $k_{e l}$, convergence rates were $89.6 \%$ for LS design and $76.8 \%$ for DS design.

The major limitation of the present study is that, numerical values (e.g. 95\% range of \%bias) obtained from simulation studies are case-specific and thus an evaluation for each individual cases is needed. For example, the lower and upper limits of the 95\% range of \%bias of the population mean $k_{e l}$ estimated for simulated data sets from the LS design were $-40 \%$ and $50 \%$, respectively. These values depend on the model parameters that simulate the datasets. There is still room to examine how 
the simulated data should be added to the real data. This includes the time points to obtain observations, the number of observations per subject, and the number of subjects.

\section{Conclusion}

We assert that the reliability of estimated values of PPK parameters can be evaluated by stochastic simulation and estimation in analysis of data obtained in the LS design. Subsequently, we proposed the method that estimates the direction and magnitude of the bias for any parameters that have problems with precision or accuracy by using historical knowledge. This study should stimulate ideas for alternative strategies to estimate PPK parameters from limited data, by highlighting the problems associated with analysis of data from limited sampling designs.

\section{References}

[1] Gabrielsson, J.; Weiner, D. Pharmacokinetic and Pharmacodynamic Data Analysis: Concepts and Applications, 4th ed.; Swedish Pharmaceutical Press: Stockholm, 2000; pp 11-224.

[2] Beal, S. L.; Sheiner, L. B. Estimating population kinetics. Crit. Rev. Biomed. Eng. 1982, 8, 195-222.

[3] Aarons, L. Population pharmacokinetics: theory and practice. British Journal of clinical pharmacology 1991, 32, 669-670.

[4] Aarons, L. Population pharmacokinetics. International Journal of Clinical Pharmacology, Therapy, and Toxicology 1992, 30, 520-522.

[5] Mandema, J. W.; Verotta, D.; Sheiner, L. B. Building population pharmacokinetic-pharmacodynamic models. I. Models for covariate effects. J. Pharmacokinet. Biopharm. 1992, 20, 511-528.

[6] Sheiner, L. B.; Beal, S. L. Evaluation of methods for estimating population pharmacokinetic parameters. III. Monoexponential model: routine clinical pharmacokinetic data. $J$. Pharmacokinet. Biopharm. 1983, 11, 303-319.

[7] Al-Banna, M. K.; Kelman, A. W.; Whiting, B. Experimental design and efficient parameter estimation in population pharmacokinetics. J. Pharmacokinet. Biopharm. 1990, 18, 347-360.

[8] Jonsson, E. N.; Wade, J. R.; Karlsson, M. O. Comparison of some practical sampling strategies for population pharmacokinetic studies. J. Pharmacokinet. Biopharm. 1996, 24, 245-263.

[9] Ette, E. I.; Sun, H.; Ludden, T. M. Balanced designs in longitudinal population pharmacokinetic studies. J. Clin. Pharmacol. 1998, 38, 417-423.

[10] Kowalski, K. G.; Hutmacher, M. M. Design evaluation for a population pharmacokinetic study using clinical trial simulations: a case study. Stat. Med. 2001, 20, 75-91.

[11] Roy, A.; Ette, E. I. A pragmatic approach to the design of population pharmacokinetic studies. AAPS J. 2005, 7, E408-420.

[12] Klein, K.; Gueorguieva, I.; Aarons, L. Population pharmacokinetic modelling of S-warfarin to evaluate the design of drug-drug interaction studies for CYP2C9. Journal of Pharmacokinetics and Pharmacodynamics 2012, 39, 147-160. 
[13] van Hasselt, J. G.; van Eijkelenburg, N. K.; Beijnen, J. H.; Schellens, J. H.; Huitema A. D. Design of a drug-drug interaction study of vincristine with azole antifungals in pediatric cancer patients using clinical trial simulation. Pediatric Blood \& Cancer 2014, 61, 2223-2239.

[14] Ogungbenro, K.; Hulme, S.; Rothwell, N.; Hopkins, S.; Tyrrell, P.; Galea, J. Study design and population pharmacokinetic analysis of a phase II dose-ranging study of interleukin-1 receptor antagonist. Journal of Pharmacokinetics and Pharmacodynamics 2016, 43, 1-12.

[15] Mentré, F.; Mallet, A.; Baccar, D. Optimal design in random-effects regression models. Biometrika 1997, 84, 429-442.

[16] Duffull, S. B.; Retout, S.; Mentré, F. The use of simulated annealing for finding optimal population designs. Computer Methods and Programs in Biomedicine 2002, 69, 25-35.

[17] Nyberg, J.; Bazzoli, C.; Ogungbenro, K.; Aliev, A.; Leonov, S.; et al. Methods and software tools for design evaluation in population pharmacokinetics-pharmacodynamics studies. British Journal of Clinical Pharmacology 2015, 79, 6-17.

[18] Bonate, P. L. Pharmacokinetic-pharmacodynamic modeling and simulation, Springer: London, 2011; pp 330-336.

[19] Meibohm, B.; Läer, S.; Panetta, J. C.; Barrett, J. S. Population pharmacokinetic studies in pediatrics: issues in design and analysis. AAPS J. 2005, 7, E475-487.

[20] Bortot, P.; Thomaseth, K.; Salvan, A. Population toxicokinetic analysis of 2,3,7,8tetrachlorodibenzo-p-dioxin using Bayesian techniques. Statistics in Medicine 2002, 21, 533 547.

[21] Gisleskog, P. O.; Karlsson, M. O.; Beal, S. L. Use of prior information to stabilize a population data analysis. J. Pharmacokinet. Pharmacodyn. 2002, 29, 473-505.

[22] Ribbing, J.; Hooker, A. C.; Jonsson, E. N. Non-Bayesian knowledge propagation using modelbased analysis of data from multiple clinical studies. J. Pharmacokinet. Pharmacodyn. 2008, 35, 117-137.

[23] Nemoto, A. Study of pharmacokinetic analysis of blood alcohol concentration and data sampling design. Master's thesis in Tokyo University of Science, 2008.

[24] Jones, A. W.; Jönsson, K. A. Food-induced lowering of blood-ethanol profiles and increased rate of elimination immediately after a meal. J. Forensic. Sci. 1994, 39, 1084-1093.

[25] Norberg. A.; Jones, A. W.; Hahn, R. G.; Gabrielsson, J. L. Role of variability in explaining ethanol pharmacokinetics: research and forensic applications. Clin. Pharmacokinet. 2003, 42, $1-31$.

[26] Widmark, E. M. P. Principles and applications of medicolegal alcohol determination, Biomedical publications: Davis, 1981.

[27] Yamamoto, K.; Ueno, Y.; Mizoi, Y.; Tatsuno, Y. Genetic polymorphism of alcohol and aldehyde dehydrogenase and the effects on alcohol metabolism. Arukoru Kenkyuto Yakubutsu Ison 1993, 28, 13-25.

[28] Gullberg, R. G.; Jones, A. W. Guidelines for estimating the amount of alcohol consumed from a single measurement of blood alcohol concentration: re-evaluation of Widmark's equation. Forensic. Sci. Int. 1994, 69, 119-130.

[29] Gullberg, R. G. Estimating the uncertainty associated with Widmark's equation as commonly applied in forensic toxicology. Forensic. Sci. Int. 2007, 172, 33-39.

[30] Nishimura, F. T.; Kimura, Y.; Abe, S.; Fukunaga, T.; Saijoh, K. Effect of -361 G/A polymorphism of aldehyde dehydrogenase-2 gene on alcohol metabolism and its expression in human peripheral blood leukocytes. Nihon Arukoru Yakubutsu Igakkai Zasshi 2006, 41, 108119.

[31] Dettling, A.; Fischer, F.; Böhler, S.; Ulrichs, F.; Skopp, G.; et al. Ethanol elimination rates in men and women in consideration of the calculated liver weight. Alcohol 2007, 41, 415-420. 
[32] Ette, I. E.; Roy, A. Pharmacometrics: The science of quantitative pharmacology, Ette, I. E.; Williams, P. J.; Eds.; Wiley: New Jersey, 2001; pp 303-344.

[33] Wakefield, J. The Bayesian analysis of population pharmacokinetic models. Journal of the American Statistical Association 1996, 91, 62-75.

[34] Norberg, Å.; Gabrïelsson, J.; Jones, A. W.; Hahn, R. G. Within- and between-subject variations in pharmacokinetic parameters of ethanol by analysis of breath, venous blood and urine. $B r . J$. Clin. Pharmacol. 2000, 49, 399-408.

[35] Lee, B. Y.; Yoon, H. K.; Baek, I. H.; Kwon, K. I. Population pharmacokinetics of multiple alcohol intake in humans. Alcohol 2013, 47, 159-165.

[36] U. S. Food and Drug Administration/Center for Drug Evaluation and Research and Center for Biologics Evaluation and Research, Guidance for Industry: Population Pharmacokinetics, 1999. http://www.fda.gov/downloads/Drugs/Guidances/UCM072137.pdf (accessed Oct 25, 2015). 